Case Report

\title{
Rupture of Hepatocellular Carcinoma after Transarterial Chemoembolization followed by Massive Gastric Bleeding
}

\author{
Kazuhiro Nishida $\left(\mathbb{D},{ }^{1}\right.$ Alan Kawarai Lefor, ${ }^{2}$ and Tomohiro Funabiki ${ }^{1}$ \\ ${ }^{1}$ Department of Emergent and Critical Care Medicine, Saiseikai Yokohamashi Tobu Hospital, 3-6-1, Shimosueyoshi, Tsurumi, \\ Yokohama-city, Kanagawa 230-8765, Japan \\ ${ }^{2}$ Department of Surgery, Jichi Medical University, 3311-1 Yakushiji, Shimotsuke, Tochigi 329-0498, Japan
}

Correspondence should be addressed to Kazuhiro Nishida; pandadebanda4@gmail.com

Received 28 February 2018; Accepted 22 April 2018; Published 4 June 2018

Academic Editor: Dario Lorenzin

Copyright (C) 2018 Kazuhiro Nishida et al. This is an open access article distributed under the Creative Commons Attribution License, which permits unrestricted use, distribution, and reproduction in any medium, provided the original work is properly cited.

\begin{abstract}
Introduction. Transarterial chemoembolization (TACE) is the first-line therapy for patient with unresectable hepatocellular carcinoma (HCC). Although TACE is a generally safe procedure, major complications can be occurred. We describe a patient with rupture of HCC after TACE followed by gastric bleeding. Case Presentation. An 81-year-old man presented with worsening epigastric pain. He had been diagnosed with multiple HCC with nonalcoholic steatohepatitis and underwent TACE 19 days previously. A contrast enhanced computed tomography (CT) scan of the abdomen showed rupture of an HCC. He was treated nonoperatively and discharged on hospital day 18. Five weeks after TACE, he was emergently admitted with massive hematochezia and shock. A contrast enhanced CT scan demonstrated extrinsic gastric compression by an HCC lesion with extravasation of contrast into the stomach. Emergent upper gastrointestinal endoscopy showed a bleeding gastric ulcer with extraluminal compression which was successfully controlled by hypertonic saline-epinephrine injection. Due to tumor progression, he was discharged for palliative care and died six weeks after TACE. Conclusion. Rupture of HCC is a life-threatening complication after TACE with mortality rates up to $50 \%$. After treatment of a ruptured HCC, extragastric compression and bleeding can occur due to direct compression by a primary lesion or intraperitoneal dissemination.
\end{abstract}

\section{Background}

Transarterial chemoembolization (TACE) is the first-line therapy for patients with an unresectable hepatocellular carcinoma (HCC) and hepatic metastases. Although TACE is a generally safe, major complications such as tumor rupture, liver abscess, bile leak, hepatic failure, gastrointestinal hemorrhage/ulceration, and pulmonary embolism can occur [1-5]. The major complication rate is reported to be $2.1-2.5 \%$ with a mortality rate up to $16.7 \%[2,4]$. Especially for the patients with an HCC larger than $10 \mathrm{~cm}$, the major complication rate is as high as $4.9 \%$ [3]. The incidence of ruptured HCC after TACE is very low, with few published reports. In the 1990s, Liu reported 391 patients who underwent 1443 sessions of TACE, with rupture of an HCC occurring in only six patients ( $1.5 \%$ per patient and $0.4 \%$ per procedure) [5]. Two decades later, Tu reported on 1120 patients who underwent 2863 sessions of TACE. Six patients suffered rupture of the
HCC ( $0.5 \%$ per patient and $0.2-0.4 \%$ per procedure) and two of them died [2]. HCC rupture after TACE is rare but can be a fetal complication.

\section{Case Presentation}

An 81-year-old man presented with abdominal discomfort and distention. His medical history was remarkable for hypertension and type 2 diabetes mellitus. He and his family denied alcohol abuse. Physical examination revealed hepatomegaly without jaundice, ascites, or hepatic encephalopathy. Laboratory data included platelet count $101,000 / \mathrm{mm}^{3}$, total bilirubin $0.9 \mathrm{mg} / \mathrm{dl}$, AST $33 \mathrm{IU} / \mathrm{L}$, ALT IU/L, and PT-INR 1.15. The serum AFP and PIVKA-II levels were $1081.0 \mathrm{ng} / \mathrm{ml}$ and $43 \mathrm{mAU} / \mathrm{ml}$. Serologic tests for hepatitis $\mathrm{B}$ and $\mathrm{C}$ virus were negative. The diagnosis of nonalcoholic steatohepatitis with Child-Pugh A liver cirrhosis was made and four HCC 


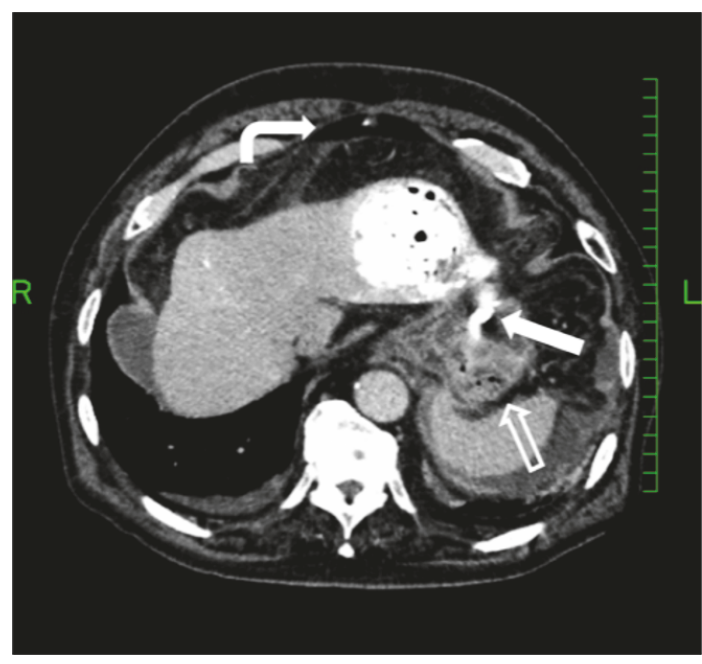

FIGURE 1: A contrast enhanced computed tomography scan of the abdomen showed iodized oil (arrow) and intraperitoneal free (curved arrow) air with a rupture of the HCC in segment II into the peritoneal cavity adjacent to the gastric wall (open arrow).

lesions were found in segments II, VI, and VIII on the imaging. The largest one was located near the liver capsule in segment II measuring $6.5 \mathrm{~cm}$ in diameter. The other three lesions were less than $2 \mathrm{~cm}$ with one in segment VI and two in segment VIII. Segmental TACE with epirubicin and iodized oil was performed and he was discharged uneventfully.

He was doing well until he developed abdominal pain 15 days after TACE which was gradually getting worse emergency transport to the hospital. His temperature was $37.7^{\circ} \mathrm{C}$, blood pressure 102/41 $\mathrm{mmHg}$, and pulse rate 79/minute. On physical examination, the abdomen was distended and hard to palpation without rebound tenderness. His hemoglobin was $12.6 \mathrm{~g} / \mathrm{dl}$. A contrast enhanced computed tomography (CT) scan of the abdomen showed iodized oil and intraperitoneal free air with a rupture of the HCC in segment II into the peritoneal cavity adjacent to the gastric wall (Figure 1). Extravasation of contrast medium was not seen. Emergent upper gastrointestinal endoscopy confirmed no gastric mucosal lesions or a site of perforation. Without evidence of septic shock or hemorrhage, surgical drainage and transcatheter arterial embolization (TAE) are considered less effective. He was treated nonoperatively with piperacillin and tazobactam. Although an abdominal abscess formed, he was discharged on hospital day 18 with continued antimicrobial therapy.

Five weeks after undergoing TACE, he was readmitted with hematochezia and hemorrhagic shock. The hemoglobin level was dropped to $6.6 \mathrm{~g} / \mathrm{dl}$. A contrast enhanced CT scan demonstrated gastric extraluminal compression by an HCC lesion with extravasation of contrast medium into the stomach (Figure 2). Emergent upper gastrointestinal endoscopy showed a submucosal tumor with central ulceration located on the anterior wall of the gastric body, corresponding to extraluminal compression by a HCC (Figure 3). The hemorrhage from the ulcer was successfully controlled by
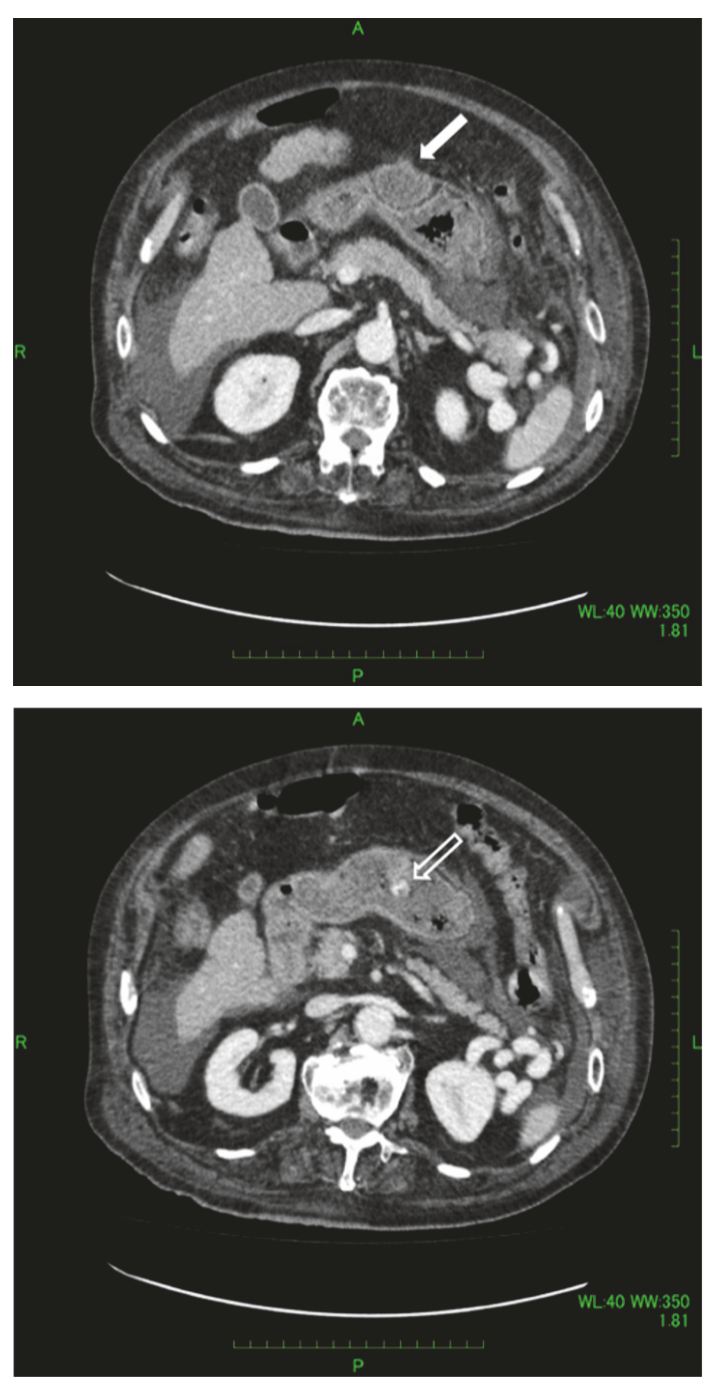

FIGURE 2: A contrast enhanced computed tomography scan demonstrated gastric extraluminal compression by hepatocellular carcinoma (arrow) and extravasation of contrast medium into the stomach (open arrow).

hypertonic saline-epinephrine injection. Another submucosal tumor was found in the gastric fundus without ulcer formation (Figure 4). The patient's condition stabilized and he was discharged for palliative care and died six weeks after undergoing TACE.

\section{Discussion}

The incidence rate of ruptured HCC after TACE is low with few cases reported. An extensive search was conducted (http://www.pubmed.com) for articles related to this topic, using the following search terms: "ruptured hepatic carcinoma" and "TACE" or "transarterial chemoembolization." A total of 21 previously reported patients were identified and are summarized in Table 1 [2, 6-16].

Fourteen patients were reported from Asia where it is known that hepatitis $C$ virus infection is relatively common. 


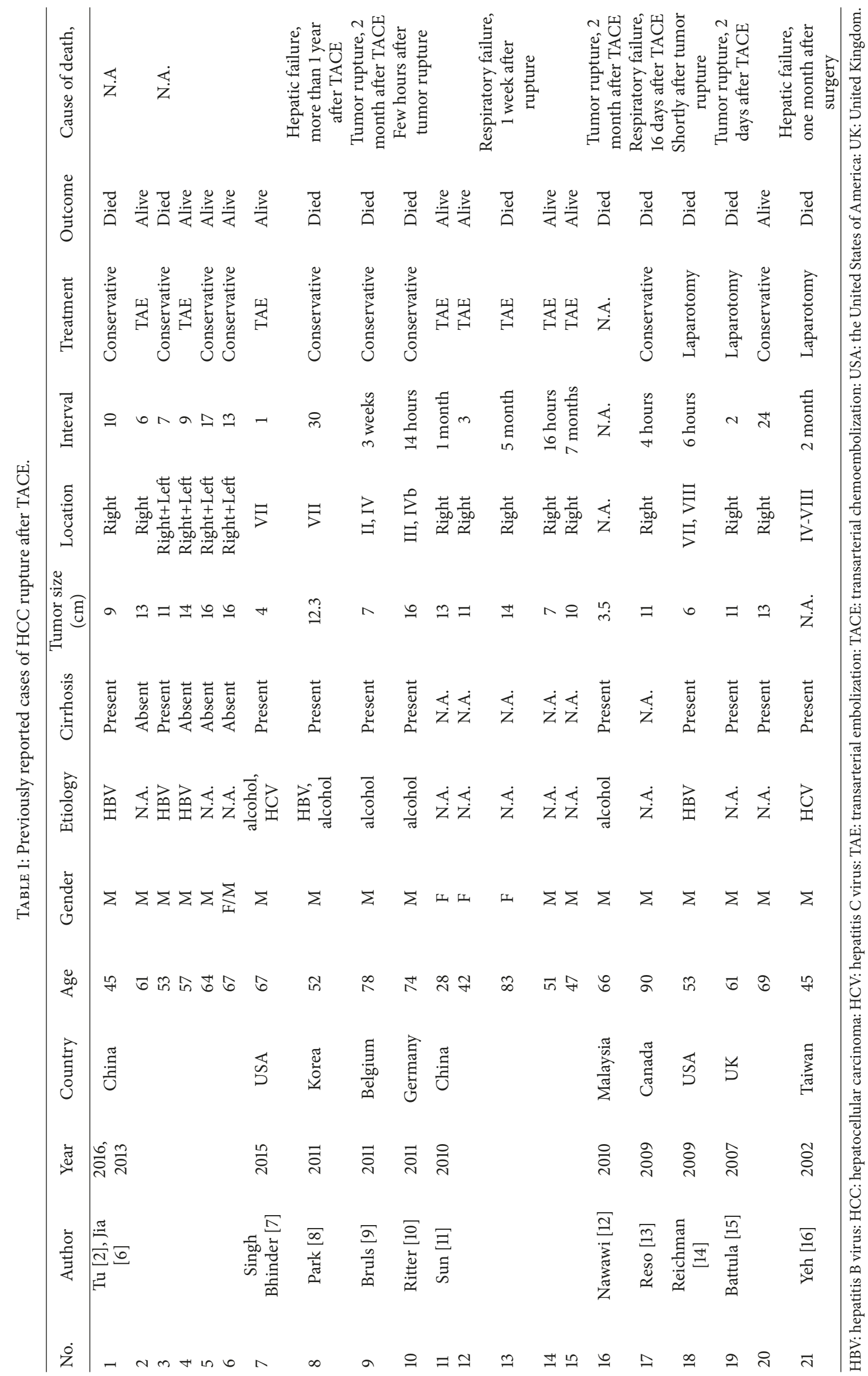




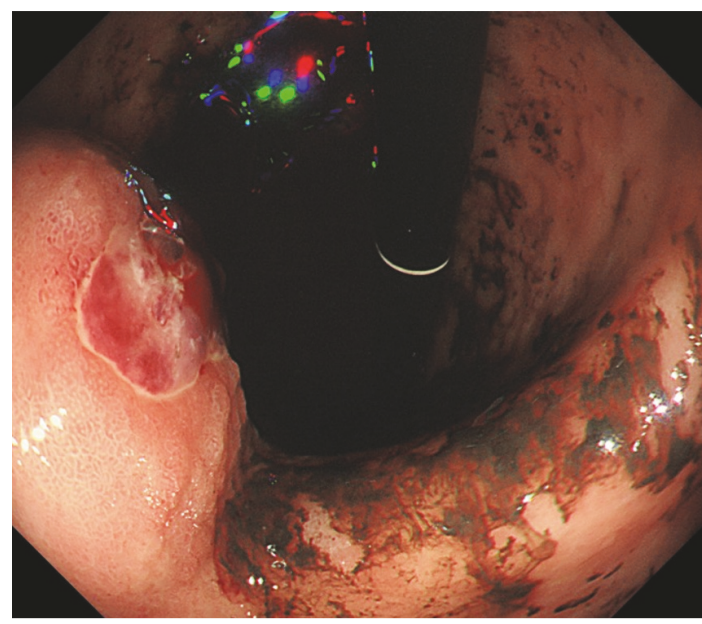

FIGURE 3: Emergent upper gastrointestinal endoscopy showed a submucosal tumor with central ulceration located on the anterior wall of the gastric body, corresponding to extraluminal compression by the hepatocellular carcinoma.

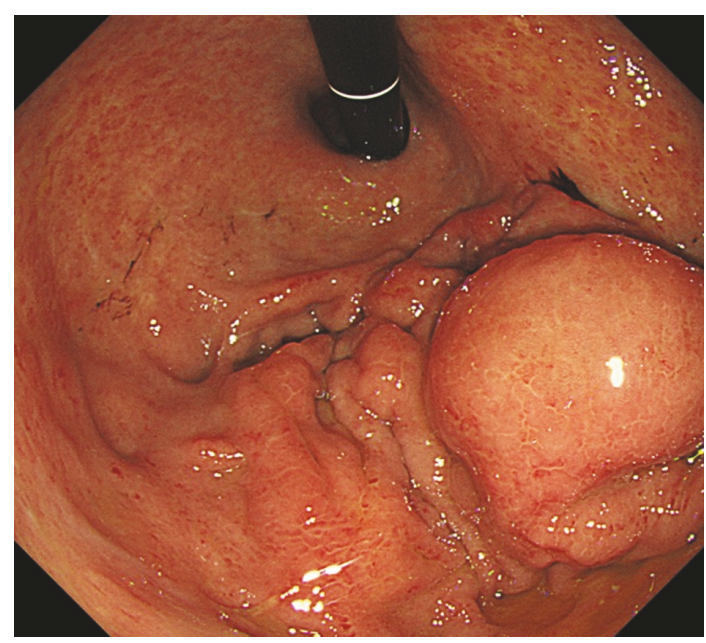

Figure 4: Another submucosal tumor was found in the gastric fundus without ulcer formation.

The age ranges from 28 to 90 with a mean of 60 years old. Male gender was $17 / 20$, except for one patient (Number 6 in Table 1) reported as a female by $\mathrm{Tu}$ and as a male by Jia [2, 6]. Fourteen of 20 patients had a tumor over $10 \mathrm{~cm}$ in diameter. Some case reports suggest that a large tumors increase the risk of rupture $[2,6,7,15]$. The cause of rupture after TACE is unknown, but fragility of the tumor wall and increased intratumoral pressure are thought to contribute to this complication. Occlusion of the feeding artery leads to necrosis of the tumor which results in wall fragility. Necrosis also leads to increased intratumoral pressure and secondary infection, especially by anaerobic gas forming organisms. In the present patient, the tumor size was $6.5 \mathrm{~cm}$ but located near the liver surface. Thin normal liver parenchyma might easily rupture if the tumor wall becomes necrotic with an increase in the intratumoral pressure. An anaerobic infection may have played an important role in the rupture because gas was seen in the tumor and intraperitoneally on the CT obtained at admission in the same location that an abscess later formed.

Treatment for rupture of an HCC after TACE rupture is either nonoperative or exploratory laparotomy. In the last 20 years, TAE has been increasingly used to control bleeding. If there are no signs of peritonitis or active bleeding; nonoperative therapy may be the treatment of choice. If concomitant hemorrhage is evident and hemostasis is necessary, TAE may be better tolerated than laparotomy. The interval between TACE and rupture has been reported 4-6 hours to 5-7 months. A shorter interval seemed to be associated with shorter survival. Since the mortality rate reached $52.4 \%$ $(11 / 21)$ in previously reported patients who suffered HCC rupture, this is a high mortality complication after TACE.

None of the patients with a ruptured HCC had gastric compression or gastrointestinal bleeding except for the present patient. Generally, extragastric compression is rare. Chen et al. performed endoscopic ultrasonography on 55 patients with extragastric compression and found five with malignant etiologies, one of which was HCC [17]. Direct compression by a primary lesion may have resulted in gastric wall extension with erosion which progressed to bleeding.

\section{Conclusion}

HCC rupture is a life-threatening complication after TACE with a mortality rate up to $50 \%$. After treatment of HCC rupture, extragastric compression with subsequent gastric bleeding can occur due to direct compression by a primary lesion or disseminated disease.

\section{Consent}

Written informed consent was obtained from the patient for publication of this case report and any accompanying images.

\section{Conflicts of Interest}

The authors declare they have no conflicts of interest.

\section{Authors' Contributions}

Kazuhiro Nishida gathered patient's date, designed the case report, and drafted manuscript. Alan Kawarai Lefor supervised the report. Tomohiro Funabiki conceived of the study and participated in its design and coordination. All authors read and approved the final manuscript.

\section{References}

[1] A. Basile, G. Carrafiello, A. M. Ierardi, D. Tsetis, and E. Brountzos, "Quality-improvement guidelines for hepatic transarterial chemoembolization," CardioVascular and Interventional Radiology, vol. 35, no. 4, pp. 765-774, 2012.

[2] J. Tu, Z. Jia, X. Ying et al., "The incidence and outcome of major complication following conventional TAE/TACE for hepatocellular carcinoma," Medicine (United States), vol. 95, no. 49, p. e5606, 2016. 
[3] T. Xue, F. Le, R. Chen et al., "Transarterial chemoembolization for huge hepatocellular carcinoma with diameter over ten centimeters: a large cohort study," Medical Oncology, vol. 32, no. 3, 2015.

[4] J. Xia, Z. Ren, S. Ye et al., "Study of severe and rare complications of transarterial chemoembolization (TACE) for liver cancer," European Journal of Radiology, vol. 59, no. 3, pp. 407-412, 2006.

[5] C.-L. Liu, H. Ngan, C. M. Lo, and S. T. Fan, "Ruptured hepatocellular carcinoma as a complication of transarterial oily chemoembolization," British Journal of Surgery, vol. 85, no. 4, pp. 512-514, 1998.

[6] Z. Jia, F. Tian, and G. Jiang, "Ruptured hepatic carcinoma after transcatheter arterial chemoembolization," Current Therapeutic Research, Clinical and Experimental, vol. 74, pp. 41-43, 2013.

[7] N. Singh Bhinder and S. M. Zangan, "Hepatocellular carcinoma rupture following transarterial chemoembolization," Seminars in Interventional Radiology, vol. 32, no. 1, pp. 49-53, 2015.

[8] Y. H. Park, S. H. Kang, S. U. Kim et al., "A Case of Hepaticoduodenal Fistula Development after Transarterial Chemoembolization in Patient with Hepatocellular Carcinoma," The Korean Journal of Gastroenterology, vol. 58, no. 3, pp. 149-152, 2011.

[9] S. Bruls, J. Joskin, R. Chauveau, J. Delwaide, and P. Meunier, "Ruptured hepatocellular carcinoma following trans catheter arterial chemoembolization," Journal Belge de Radiologie Belgisch Tijdschrift voor Radiologi, vol. 94, no. 2, pp. 68-70, 2011.

[10] C. O. Ritter, M. Wartenberg, A. Mottok et al., "Spontaneous liver rupture after treatment with drug-eluting beads," CardioVascular and Interventional Radiology, vol. 35, no. 1, pp. 198-202, 2012.

[11] JH. Sun, LG. Wang, HW. Bao, JL. Lou, LX. Cai, and C. Wu, "Emergency embolization in the treatment of ruptured hepatocellular carcinoma following transcatheter arterial chemoembolization," Hepatogastroenterology, vol. 201, no. 57, pp. 616-619.

[12] O. Nawawi, M. Hazman, B. Abdullah et al., "Transarterial embolisation of hepatocellular carcinoma with doxorubicineluting beads: single centre early experience," Biomedical Imaging and Intervention Journal, vol. 6, no. 1, 2010.

[13] A. Reso, C. G. Ball, F. R. Sutherland, O. Bathe, and E. Dixon, "Rupture and intra-peritoneal bleeding of a hepatocellular carcinoma after a transarterial chemoembolization procedure: A case report," Cases Journal, vol. 2, no. 1, 2009.

[14] T. W. Reichman, T. Anthony, J. M. Millis, and G. Testa, "Uncharacteristically early fatal intraperitoneal rupture of hepatocellular carcinoma following transarterial chemoembolization," Digestive and Liver Disease, vol. 41, no. 2, pp. 175-176, 2009.

[15] N. Battula, M. Madanur, O. Priest et al., "Spontaneous rupture of hepatocellular carcinoma: a Western experience," The American Journal of Surgery, vol. 197, no. 2, pp. 164-167, 2009.

[16] C.-N. Yeh, H.-M. Chen, M.-F. Chen, and T.-C. Chao, "Peritoneal implanted hepatocellular carcinoma with rupture after TACE presented as acute appendicitis," Hepato-Gastroenterology, vol. 49, no. 46, pp. 938-940, 2002.

[17] T. K. Chen, C. H Wu, C. L. Lee, Y. C. Lai, S. S. Yang, and T. C. Tu, "Endoscopic ultrasonography to study the causes of extragastric compression mimicking gastric submucosal tumor," J Formos Med Assoc, vol. 100, pp. 100-758, 2001. 


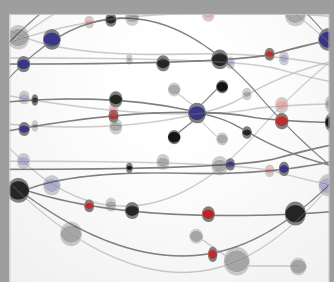

The Scientific World Journal
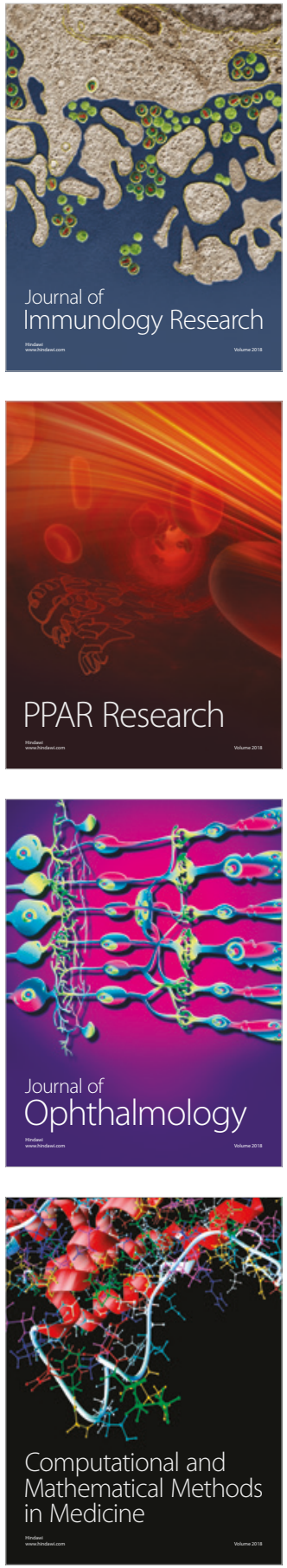

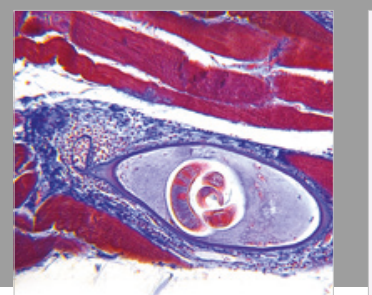

Gastroenterology Research and Practice

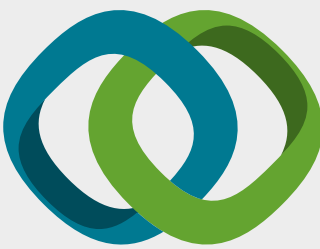

\section{Hindawi}

Submit your manuscripts at

www.hindawi.com
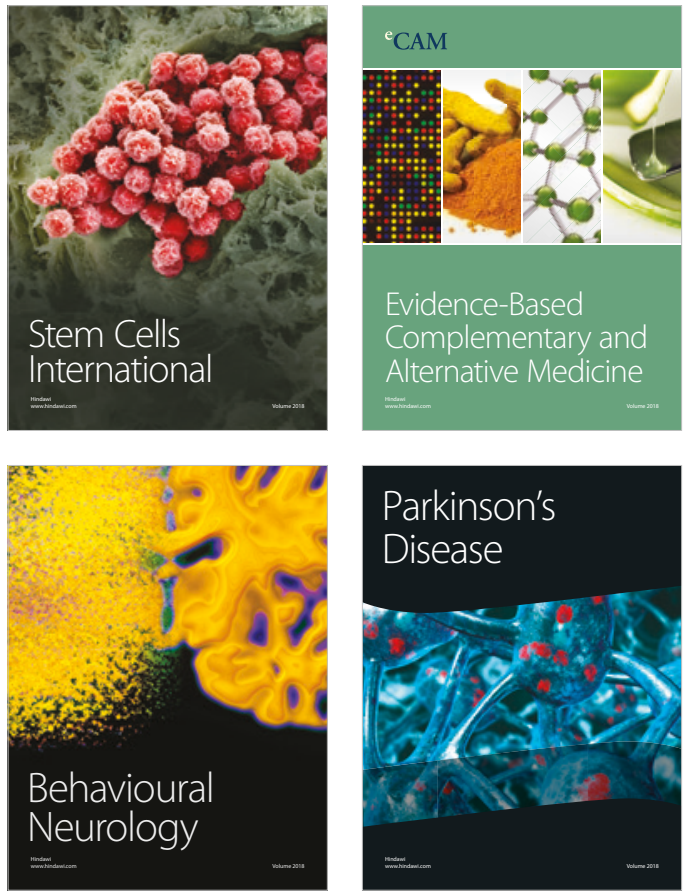

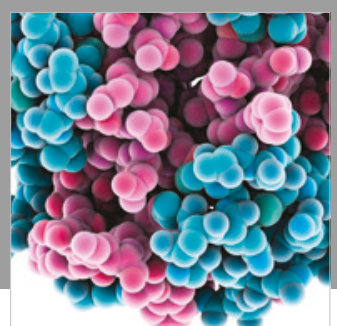

ournal of

Diabetes Research

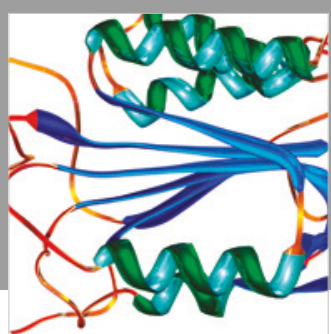

Disease Markers
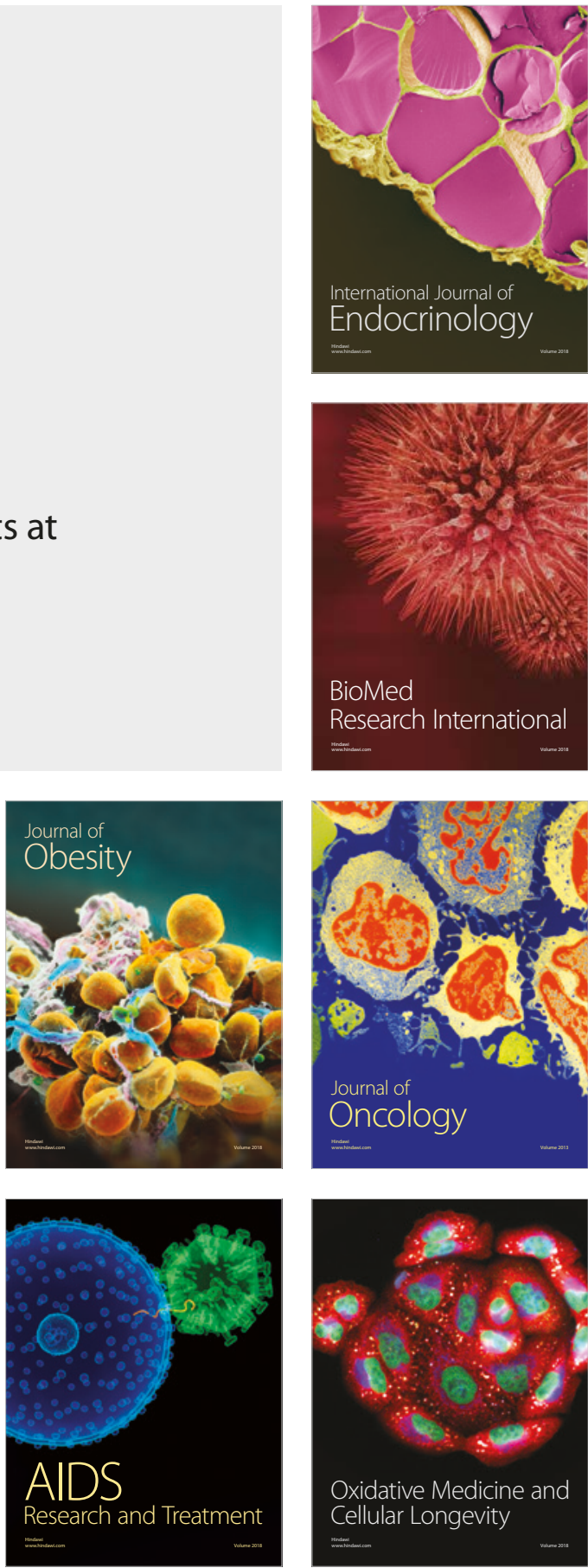